\title{
Improving the preoperative care of patients with femoral neck fractures through the development and implementation of a checklist
}

\author{
Riaz Agha, Eric Edison, Alexander Fowler \\ Addenbrookes Hospital,Cambridge University Hospitals NHS Foundation Trust
}

\begin{abstract}
The incidence of femoral neck fractures (FNFs) is expected to rise with life expectancy. It is important to improve the safety of these patients whilst under the care of orthopaedic teams.
\end{abstract}

This study aimed to increase the performance of vital preoperative tasks in patients admitted for femoral neck fracture operations by producing and implementing a checklist as an aide memoir. The checklist was designed primarily for use by senior house officers (SHOs) admitting patients from the emergency department.

A list of 12 preoperative tasks was identified. A baseline audit of 10 random patients showed that the mean proportion of the 12 tasks completed was $53 \%$ (range $25 \%-83 \%$ ). A survey of 14 nurses and surgeons found that the majority of respondents agreed that there was a problem with the performance of most of the tasks. The tasks were incorporated into a checklist which was refined in three plan-do-study-act cycles and introduced into the femoral neck fracture pathway.

In the week following the introduction of the checklist, $77 \%$ of the checklist tasks were completed, $24 \%$ more than at the baseline audit (53\%). In week 3 , the completion of checklist tasks rose to $88 \%$ and to $95 \%$ in week 4 .

In conclusion, a simple checklist can markedly improve the performance and recording of preoperative tasks by SHOs. We recommend the wider adoption of the new checklist to be produced as a sticker for patients' medical records. Further study is required to ascertain the effect of the checklist on clinical outcomes.

\section{Problem}

Patients admitted through the emergency department with FNFs and requiring an operation need a range of tests and procedures to optimise their preoperative condition. These procedures include marking the intended surgical-site to reduce the risk of wrong-site operations. (10) Also, the intended procedure should be fully explained to the patient prior to the seeking of informed consent to proceed. An ECG reading should be obtained for men over 40 and women over 50 years of age, to identify underlying cardiac comorbidities, as these may increase the patient's operative risk. If not already known, the patient's blood group should be ascertained and serum saved should a transfusion be needed. Patients' venous thromboembolism (VTE) risk should be assessed and appropriate prophylaxis prescribed to reduce this risk. Some of these tasks are absolute requirements as defined by the WHO's surgical safety checklist.

The lead author (RA) identified several local factors which had the potential to lead to the non-performance of some of these tasks, and therefore compromise patient safety. These include an increase in the ratio of consultants to senior house officers (SHOs) which has led to increased demands on $\mathrm{SHOs}$ who are caring for more patients preoperatively. This can potentially increase the risk of errors of omission. For example, the requirement that an ECG is not just performed but is also signed off by a doctor may be unmet because a busy SHO, without reminding, may have performed and read an ECG but forgotten to sign and document this in the patient's notes.

\section{Background}

An estimated 234 million operations are performed globally each year. (1) This amount of intervention, combined with surgery's classification as a "very unsafe industry" (2) has made it imperative that methods of improving patient safety are explored. Current initiatives include the World Health Organisation's (WHO) surgical safety checklist which acts as an aide memoir of certain vital tasks to be completed before, during, and after every operation. (3) There are several examples of other checklists which have been shown to improve the quality of care delivered to surgical patients. $(4,5)$

In the United Kingdom (UK), 32.6\% of three million surgical patient safety incidents reported to the National Patient Safety Agency were related to the trauma and orthopaedics specialty. (6) It is important to recognise that the risks of any operation are not solely due to technical challenges, but may also be compounded by the patient's preoperative condition among other factors. For example, femoral neck fractures (FNFs) are a leading cause of morbidity and mortality in elderly patients. The mortality rate of patients with FNFs is approximately $10 \%$ at 1 month and $30 \%$ at one year with a total cost to the National Health Service (NHS) of approximately £1.4 
billion annually. (7) The incidence of FNFs is projected to be 6.26 million by the year 2050, compared to 1.66 million in 1990. (8) The risks associated with treating these fractures are compounded by the comorbidities usually suffered by elderly patients. The Hip Fracture Perioperative network make a number of recommendations for optimal perioperative practice. (9) Patients with any such co-morbidity should be identified preoperatively as such patients require careful management to reduce operative risk.

The study took place at Addenbrookes Hospital, a part of the Cambridge University Hospitals NHS Foundation Trust. The hospital is a tertiary referral centre for a number of specialties and has a total of 943 inpatient beds. In 2010, the ratio of the number of in-hospital deaths for FNFs to the expected number of deaths at Addenbrookes Hospital was slightly over half the national average (53.81 vs. 100). (9)

The aim of this study was to increase the uniformity and quality of the preoperative care provided by SHOs to patients admitted for FNF surgical treatment. It was suggested that a checklist containing all the requisite preoperative tasks could potentially lead to such standardisation of care. The key target was ensuring that by the fourth week after the introduction of the checklist, all patients admitted for FNF operations from the emergency department had all checklist tasks completed preoperatively.

\section{Baseline Measurement}

To determine the extent of the local problem, six band 6 nurses, four registrars (residents), and four consultant orthopaedic surgeons were surveyed on their thoughts regarding the adequacy of several aspects of preoperative care using a questionnaire (appendix 2).

A baseline audit of 10 patients was also carried out to ascertain the proportion of patients in whom the tasks identified in the checklist were being met before any interventions.

The baseline audit of 10 patients, chosen at random, showed that the mean number of checklist tasks completed was $6.4(53 \%)$ but varied widely from $3-10(25 \%-83 \%)$ (figure 1$)$. The variation suggests that the care being provided by SHOs was not uniform. These results were concordant with the findings of the survey of nurses and consultants, the majority of whom generally agreed that there was a problem with preoperative patient marking, consenting, blood test results recording, grouping and saving of blood, completion of drug charts, and keeping patients nil by mouth (table 1).

See supplementary file: ds2338.docx - "Table 1 and Figure 1."

\section{Design}

After consulting SHOs and advanced nurse practitioners (ANP), the safer orthopaedic surgery checklist (SOSC) was designed (appendix 1). A teaching session was held to introduce the first draft of the checklist to the SHOs and ANPs. The SOSC was to be completed by the SHOs pre-operatively preparing patients for surgery and stored in the patients' medical records. A preoperative task was deemed met if the procedure was performed before midday the day after admission.

\section{Strategy}

Completion rates of the SOSC were observed weekly and plan-dostudy-act (PDSA) cycles were completed at each weekly assessment. In week 1 , the baseline audit was performed as described above. In week 2 the SOSC was introduced and tested by on-call SHOs on four patients for two days. Feedback from an orthopaedic surgeon and other SHOs was that although the checklist was relatively easy to use, the wording and design could be improved. These recommendations were accepted and the checklist was revised in accordance with the feedback. The revised checklist was again tested by on-call SHOs on four patients for two days during week 3 . A final observation of the completion of the checklist was carried out in week 4 , after which it was refined and made into a sticker to be affixed onto the patient's record.

\section{Results}

In the week following the introduction of the checklist, a mean of 9.2 $(77 \%)$ checklist tasks were completed, $24 \%$ more than at the baseline audit (figure 2). In week 3 , the completion of checklist tasks rose to $10.6(88 \%)$ and to $11.4(95 \%)$ in week 4

See supplementary file: ds2336.docx - "Figure 2."

\section{Lessons and Limitations}

Structured checklists have been used in a wide range of specialties including dermatological surgery, interventional radiology, obstetrics, and otorhinolaryngology. (12-15) Although there is a paucity of literature on the use of checklists in orthopaedics, the idea is not new. A previous study suggested that a simple checklist could be a useful way of reducing infections in arthroplasty surgery. (16)

Overall, the implementation of the checklist was unhindered bar some initial mild resistance to the introduction of the checklist, partly because of the perceived additional bureaucratic burden. There were some concerns from a minority of the SHOs that this was another "form filling exercise." Similar resistance was also noted by Sewell et al. in their study where, initially, $55 \%$ of staff thought a new checklist would cause unnecessary time delays. (11) However, when they resurveyed staff after the introduction of the checklist and staff education, $68 \%$ thought the checklist did not cause additional time delays. Staff became positive about the checklist with $68 \%$ agreeing that it improved patient safety, $77 \%$ agreeing that it improved team communication and teamwork, and $80 \%$ wanting the checklist used if they were having an operation. Similarly, one year after the introduction of a checklist in a German hospital, there was a noted improvement in interdisciplinary team building, additionally staff felt an increased sense of responsibility. 
BMJ Quality Improvement Reports

These studies and this additional data indicate the importance, when designing clinical checklists, to seek the contribution of staff that will ultimately use them. Incorporating the feedback from SHOs and ANPs into the refinement of the checklist at every PDSA step partly contributed to the week-on-week increase in completion rates (figure 2). For example, the checklist was refined to use tick boxes that took less than a minute to complete fully, this reduced the amount of time and effort required to complete the questionnaire. A completion rate of $95 \%$ in week 4 suggests that the improvements in checklist design and ease of use had been welcomed by the SHOs. The checklist was designed with SHOs in mind but is not limited to them, and could be completed by SHOs or ANPs working in the emergency department to reduce the workload for orthopaedic team members.

This quality improvement study is limited by the small number of patients used to test the checklist. However, our checklist was adopted by the orthopaedic department at Worcester Hospital and introduced into their NOF fracture pathway. (18) A baseline audit was performed to ascertain the level of adherence to the five standards in table 2 before the introduction of the SOSC. They found that only $1(10 \%)$ patient had the results of their full blood count, urea and electrolytes, and group and save documented in their notes at the time of the first audit. At the same time, $60 \%$ of men aged over 40 and women aged over 50 had been investigated by ECG and signed off by a doctor. No patients had a fullycompleted drug chart i.e. one noting their regular medications, analgesia, allergies, enoxaparin, and antibiotics. $90 \%$ of the patients were "nil by mouth" and had intravenous fluids prescribed. However, after the SOSC was introduced, they found that all the standards were being met with $100 \%$ adherence in all 10 patients examined. These results suggest that this checklist may be transferable to other hospitals, strengthening the findings from the present study.

Another limitation is that the effect of increased checklist compliance on clinical outcomes was not studied. However, the focus of this initial study was on the development of a user-friendly checklist in the first instance, with a longer follow-up study to be initiated subsequently. Although it remains unclear whether it is the completion of checklists in itself or the increased awareness of patient safety issues when checklists are introduced which benefits patients, there is some evidence that the latter is true. (19) Regardless, any initiative which improves patient safety should be embraced and examined further. Additionally, there was no reassessment of the views of the ANPs and surgeons after the introduction of the checklist because the rates of checklist completion provided an objective measure of this.

\section{Conclusion}

This study showed that a simple checklist could markedly improve the performance and recording of preoperative tasks. The checklist is being analysed to assess its suitability for use in other patient groups. We recommend the wider adoption of the SOSC to be produced as a sticker for FNF patients' medical records. Further study is required to ascertain the effect of the checklist on clinical outcomes such as surgical site infections and mortality rates.

\section{References}

1. Pace G, Carmignani L. Checklists: Are really necessary in the routinely clinical practice? Int J Surg. 2012;10(3):169-70.

2. Panesar SS, Noble DJ, Mirza SB, Patel B, Mann B, Emerton $\mathrm{M}$, Cleary $\mathrm{K}$, et al. Can the surgical checklist reduce the risk of wrong site surgery in orthopaedics?--Can the checklist help? Supporting evidence from analysis of a national patient incident reporting system. J Orthop Surg Res. 2011;6:18.

3. Haynes AB, Weiser TG, Berry WR, Lipsitz SR, Breizat AH, Dellinger EP, Herbosa T, et al. A surgical safety checklist to reduce morbidity and mortality in a global population. $\mathrm{N}$ Engl J Med. 2009 Jan 29;360(5):491-9.

4. Nakayama DK, Lester SS, Rich DR, Weidner BC, Glenn JB, Shaker IJ. Quality improvement and patient care checklists in intrahospital transfers involving pediatric surgery patients. J Pediatr Surg. 2012 Jan;47(1):112-8.

5. Croti UA, Jenkins KJ, Braile DM. Checklist in pediatric cardiac surgery in Brazil: an useful and necessary adaptation of the Quality Improvement Collaborative International Congenital Heart Surgery in Developing Countries. Rev Bras Cir Cardiovasc. 2011 JulSep;26(3):511-5.

6. Emerton M, Panesar SS, Forrest K. Safer surgery: how a checklist can make orthopaedic surgery safer. Orthop Trauma. 2009;23(5):377-80.

7. Bryson DJ, Knapp S, Middleton RG, Faizi M, Bhansali H, Uzoigwe CE. Representation to the accident and emergency department within 1-year of a fractured neck of femur. J Orthop Surg Res. 2011;6:63.

8. Cooper C, Campion G, Melton LJ. Hip fractures in the elderly: A world-wide projection. Osteoporosis International. 1992;2(6):285-9.

9. DrForsterHealth.co.uk. Cambridge University Hospitals NHS Foundation Trust - quality report 2009/10. 2012 [cited 2012 July 9]; Available from: http://www.drfosterhealth.co.uk/qualityreports/trust.aspx? otype $=2 \& i d=9$.

10. Giles SJ, Rhodes P, Clements G, Cook GA, Hayton R, Maxwell MJ, et al. Experience of wrong site surgery and surgical marking practices among clinicians in the UK. Qual Saf Health Care. 2006 Oct;15(5):363-8.

11. Sewell M, Adebibe M, Jayakumar P, Jowett C, Kong K, Vemulapalli K, Levack B. Use of the WHO surgical safety checklist in trauma and orthopaedic patients. Int orthop. 2011;35(6):897-901.

12. Smith E, Minett E, Motley R. Structured proforma for skin surgery improves speed, clarity and accuracy of documentation and effectively implements the new World Health Organization surgical checklist. Br J Dermatol. 2011;165.

13. Papadakos N, Goh GS, Belli AM. Implementing a modified World Health Organisation surgical safety checklist for interventional radiology procedures. Cardiovasc Intervent Radiol. 2011;34.

14. Kearns RJ, Uppal V, Bonner J, Robertson J, Daniel M, McGrady EM. The introduction of a surgical safety checklist 


\section{BMJ Quality Improvement Reports}

in a tertiary referral obstetric centre. BMJ Qual Safe. $2011 ; 20(9): 818-22$.

15. Helmio P, Blomgren K, Takala A, Pauniaho SL, Takala RSK, Ikonen TS. Towards better patient safety: WHO Surgical Safety Checklist in otorhinolaryngology. Clin Otolaryngol. 2011;36(3):242-7.

16. Stefansdottir A, Robertsson O, A WD, Kiernan S, Gustafson $\mathrm{P}$, Lidgren $\mathrm{L}$. Inadequate timing of prophylactic antibiotics in orthopedic surgery. We can do better. Acta Orthop. 2009 Dec;80(6):633-8.

17. Wingenfeld C, Abbara-Czardybon M, Arbab D, Frank D. Patient safety in orthopaedics: implementation and first experience with CIRS and team time-out. Z Orthop Unfall. 2010 Sep;148(5):525-31.

18. Faulkner K. An audit of preoperative checks in fractured neck of femurs. International Forum on Quality and Safety in Health Care 2011 Apr; Amsterdam2011.

19. Van WA, Hoff RG, Van EEHL, Simmermacher RKJ, Regli LPE, Kappen TH, Kalkman CJ, et al. Effects of the introduction of the WHO "surgical safety checklist" on inhospital mortality: A cohort study. Ann Surg. 2012;255(1):44-9.

\section{Declaration of interests}

No conflicts of interest

\section{Acknowledgements}

Nigel Mabvuure, Christian F. Camm 